\title{
Germanica
}

Wir sind ja alle Flüchtlinge, Fremde. Zur Migrationserfahrung bei George Tabori

"On est tous des réfugiés, des étrangers. " L'expérience de la migration chez George Tabori

Alice Bolterauer

(2) OpenEdition

Journals

Édition électronique

URL : http://journals.openedition.org/germanica/384

DOI : 10.4000/germanica.384

ISSN : 2107-0784

Éditeur

Université de Lille

Édition imprimée

Date de publication : 1 juin 2006

Pagination : 47-62

ISBN : 2-913857-17-5

ISSN : 0984-2632

Référence électronique

Alice Bolterauer, «Wir sind ja alle Flüchtlinge, Fremde. Zur Migrationserfahrung bei George Tabori »,

Germanica [Online], 38 | 2006, Online erschienen am: 19 Februar 2010, abgerufen am 06 Oktober 2020.

URL : http://journals.openedition.org/germanica/384 ; DOI : https://doi.org/10.4000/germanica.384

Ce document a été généré automatiquement le 6 octobre 2020.

(c) Tous droits réservés 


\section{Wir sind ja alle Flüchtlinge, Fremde. Zur Migrationserfahrung bei George Tabori}

"On est tous des réfugiés, des étrangers. " L'expérience de la migration chez George Tabori

Alice Bolterauer

„Im fremdsprachigen Land wird die eigene, die Muttersprache - sonst war sie Haus und Heim, Sicherheit verbürgend, Wärme und, in ihren Grenzen, das himmlische Gefühl der Grenzenlosigkeit - zum Gefängnis, aus dem auszubrechen auch bei größter Wendigkeit und Geschicklichkeit nur schwer gelingen will. Was Gerüst war einer herrlich weitgespannten Welt, schrumpft ein zu engenden Gitterstäben."

Alfred Polgar „Der Emigrant und die Heimat “

\section{Einleitung}

George Tabori, im Mai 1914 in der österreichisch-ungarischen Doppelmonarchie geboren, Sohn einer deutsch sprechenden jüdischen Familie in Budapest, ab den 30er Jahren in England und Amerika lebend, dort auf Englisch redend, arbeitend und schreibend, seit 1971 in Deutschland seine auf Englisch geschriebenen Stücke auf Deutsch inszenierend und aufführend, dieser George Tabori, der als Kellner in Berlin, als Reporter in Istanbul, als Agent in London, als Dramatiker in New York gelebt und gearbeitet hat und der in einem Interview anlässlich seines 90. Geburtstages gemeint hat, Englisch habe er verlernt, Ungarisch spreche er nur noch mit seinem Hund und auch das Deutsche verschwinde langsam ${ }^{1}$, dieser George Tabori (resp. sein Werk) soll hier unter dem Fokus der Migrationsliteratur besprochen werden, der er aufgrund 
seiner Themen und seiner Biographie unweigerlich zugehört und deren Grenzen er doch immer wieder weit übersteigt.

2 Denn wenn die seit den späten 80er Jahren sich durchsetzende Diskussion um die Eigenständigkeit und Gleichwertigkeit der Literatur von Gastarbeiter/innen, Immigrant/innen, Emigrant/innen, Zuwanderer/innen und Flüchtlingen sich unter dem Siegel „Migrationsliteratur“ ein Forum geschaffen hat, auf dem die Texte von Migrant/innen als relevanter Beitrag zur Etablierung einer multikulturellen Gesellschaft verstanden werden, dann geschah und geschieht dies zumeist unter dem Aspekt der Erweiterung und Ergänzung eines primär nationalen Literaturverständnisses. So versteht A. Mansour Bavar die deutschsprachige Migrationsliteratur als ein „typisches Beispiel für die Relevanz der pluralistischen Sichtweise in einer sich ständig verändernden Gesellschaft ${ }^{{ }^{*} \text {, }}$, bei der es - so Gerhard Bauer - um eine „bewusste und ausdrückliche Auseinandersetzung mit den Tendenzen der Vereinnahmung wie der Verallgemeinerung “ des Fremden und der Fremden gehe ${ }^{3}$. In diesem Sinn ist auch das Werk George Taboris der Migrationsliteratur zuzurechnen allerdings auf dem Level einer Weltliteratur, die sich immer schon der Themen der Heimatlosigkeit, des Sprachverlusts, der Fremdheit angenommen hatte und hat. George Taboris Texte sind Migrationsliteratur, aber nicht Teil einer "deutschen" Migrationsliteratur, sondern einer universellen und globalen, in der die Fremdheitsund Abhängigkeitserfahrungen von Schwarzen, Indianern und Juden einander die Hand geben.

3 Heidi Rüsch hat in ihrem Buch über „Migrationsliteratur im interkulturellen Kontext“ drei Kriterien benannt, die Migrationsliteratur auszeichnen und die auch diesen Beitrag strukturell gliedern werden:

1) Migration als Erfahrungshintergrund der Autor/innen und/oder als Thema ihrer Literatur;

2) Schreiben in einer anderen als der Muttersprache und/oder in einem "fremden“ Sprach- und/oder Kulturraum;

3) Literatur als ästhetische Ausdrucksform und/oder als Mittel des Kampfes gegen Unterdrückung und Ausgrenzung ${ }^{4}$.

4 Diese drei Aspekte lassen sich in George Taboris Werk nicht nur nachweisen, sondern prägen es auch auf besonders nachdrückliche Weise.

\section{Das Thema der Migration}

„Das war New York. Die beste Stadt, in der ich je gelebt habe. Ich wohnte in der 95. Straße. [...] Jeden Morgen um 8.30 Uhr brachte ich mein Kind zur Schule in die 89. Straße, da waren drei chinesische Restaurants, der Zeitungshändler war ein Grieche, der Drugstore war deutsch. ${ }^{{ }^{5}}$ Die plurikulturelle Realität, die Tabori hier in seiner Erinnerung präsentiert und die Migration und Fremdsein impliziert, ist ein wesentlicher Bestandteil von Taboris Biographie. Als ungarischer Jude arbeitet er 1932/33 für ein Jahr in Berlin, bevor er 1935 seinem Bruder nach London folgt; während des Zweiten Weltkriegs ist er als Auslandskorrespondent der BBC auf dem Balkan und im Nahen Osten tätig (in Sofia, Istanbul, Kairo); ab 1947 lebt und arbeitet George Tabori in Amerika, zuerst in Hollywood, später in New York; seit 1971 agiert er auf deutschen Bühnen, ab 1987 auch in Wien. 
Dieser Wechsel der kulturellen Kontexte, dieses permanente Herausgerissensein aus den gewohnten Erfahrungshorizonten - aus dem, was man „Heimat“ nennen könnte bildet auch in vielen Texten Taboris den unhintergehbaren Hintergrund seiner Figuren, ihrer Traumata, ihrer Ticks und ihrer Lust. Sie leiden wie Rotgesicht in „Weisman und Rotgesicht" an ihrer Zerrissenheit zwischen einem letztlich sehr erbärmlichen Heimatgefühl und ihrer abgeschobenen Existenz in moderner Zivilisation oder sie engagieren sich wie Monsieur Y in „Die Demonstration“ gegen Ungerechtigkeit in unterschiedlichen kulturellen Kontexten. „Monsieur möchte nach Mississippi. Oder ist es Missouri, ich bin nicht sicher. Eine gute Idee, meint ihr nicht auch? Er ist zweiundneunzig. Er sollte nicht mal mehr Treppen steigen. Aber, hélas, sein Interesse an der guten Sache ist noch immer ganz prometheisch." ${ }^{\text {"E }}$ Es gibt bei Tabori kaum Figuren, die dort, wo sie sich eben befinden, zu Hause sind, die sich nicht von ihrem status quo wegsehnen oder manchmal auch danach zurücksehnen, die nicht in irgendeiner Art und Weise mit Heimatverlust, Ausgesetztsein und Marginalisierung Bekanntschaft gemacht hätten. „Die exzentrische Welt ist heute die zentrale“, zitiert P. Iyer den US-Amerikaner mexikanischer Herkunft Carlos Fuentes ${ }^{7}$. Und ganz ähnlich konstatieren Elisabeth Bronfen und Benjamin Marius, dass es heute nicht mehr darum gehe, „ob wir kulturelle Hybridität für erstrebenswert halten oder nicht, sondern einzig darum, wie wir mit ihr umgehen. “8 Die „Vertreibung aus dem Paradies“9 ist die conditio sine qua non des modernen Menschen, seine Unaufhaltsamkeit sein Schicksal.

7 Nicht zufällig sind deswegen häufig Orte des Übergangs, des nur vorübergehenden Bleibens, wie ein Obdachlosenheim („Mein Kampf“), ein Soldaten-Aufnahmelager („Pinkville“), Bahnhof und Zug („Mutters Courage“) oder eine KZ-Baracke („Die Kannibalen“), Schauplätze von Taboris Stücken. Der Mensch, der sich sesshaft machen möchte, der einen Ort der Ruhe, auch des Ausruhens sucht, wird aufgescheucht, fortgetrieben, verjagt, gejagt, verfolgt und muss sich transitorisch einrichten. Dies bildet bereits in dem frühen Roman Taboris "Tod in Port Aarif" den Erfahrungshintergrund des ungarischen Arztes Varga, der in einem transsylvanischen Dorf geboren wird, nach der Ermordung des Vaters durch den aufgebrachten Mob mit der Mutter nach Budapest geht, später zum Studium nach Wien wechselt und sich schließlich für ein Leben als Schiffsarzt, d.h. ständig unterwegs, ohne feste Verankerung, entscheidet. „Festland war gefährlich; immer kam es zu Verpflichtungen, denen er nie ganz entgehen konnte ${ }^{" 10}$

Das Festland, wußte Varga, war ein sicheres Terrain für die Einheimischen, für Leute wie ihn aber gefährlich: Anrufe mußten erwidert, Hüte gezogen, Steuern gezahlt werden, und über den Gräbern lag eine internationale Stille. Da gab es immer Stimmen, die dem Besucher - und er gefiel sich in der Rolle des Besuchers zuriefen: „Ah, da sind Sie ja!“ und „Wo waren Sie denn?“ und „Kommen Sie mich doch besuchen“. Festland bedeutete Loyalität: die Loyalität des Sklaven, der glaubt, sein eigener Herr zu sein und doch nur sein eigener Sklave ist. ${ }^{11}$

8 Varga entscheidet sich bewusst für ein Nomadendasein, das ihm Freiheit und Verantwortungslosigkeit $\mathrm{zu}$ versprechen scheint - und in der Tat oder gerade deswegen ist es eben ein längerer Aufenthalt an Land, seine Präsenz und sein ungewolltes Mitwirken an den politischen Intrigen von Port Aarif, was seinen gewaltsamen Tod bewirkt.

9 Anders als der Arzt Varga in „Tod in Port Aarif“ basiert die Migrationserfahrung in vielen anderen Texten Taboris auf keiner bewussten und freiwilligen Entscheidung, sondern wird den Figuren von außen aufgezwungen. Wie eine unterirdische Ader 
durchzieht alle Texte Taboris das Wissen um die Geschichte der Judenverfolgung, der alltäglichen Demütigungen, der periodisch auftretenden Pogrome bis zum Holocaust. „Der Odessa-Zweig wurde vor etwa zweihundert Jahren aus der Stadt gepeitscht und

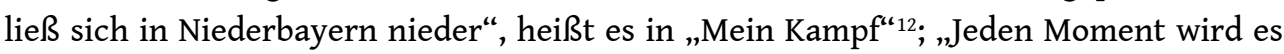
an der Tür klopfen. Dreimal vielleicht. Sie kommen mich holen. Es gibt kein Entrinnen. Dafür ist es zu spät. Holst du mir meine grüne Reisetasche? [...] Ich packe besser das Nötigste zusammen. Socken, Seife, Salami und das Alte Testament ${ }^{\text {“13 }}$, räsoniert Alfons Morgenstern in der „Ballade vom Wiener Schnitzler“ über die ewige Wiederkehr der Judenverfolgung. Und im finalen Show-down zwischen Weisman und Rotgesicht im gleichnamigen Stück wird die Erfahrung des Exils zum gewichtigen Einsatz im Streit um das größere Unglück erlittener Ungerechtigkeiten:

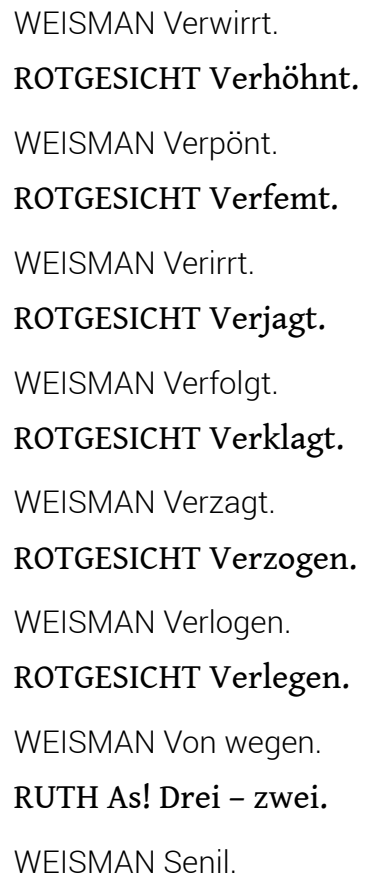

ROTGESICHT Debil.

WEISMAN Labil.

ROTGESICHT Steril.

WEISMAN Exil. ${ }^{14}$

Das Trauma der Judenverfolgung, ihrer Vertreibung und Massenvernichtung bildet den roten Faden, der sich durch alle Texte Taboris zieht $t^{15}$. Das Streben der Menschen nach Sicherheit und Geborgenheit wird sabotiert, Menschen werden aus ihren Wohnungen, aus ihren gesellschaftlichen Stellungen verjagt, Familien werden auseinandergerissen, Menschen gequält und getötet. „Wer könnte dieses schmale Skelett sein? Ach ja, Tante Gisela. Ihr Kopf wurde in zwei Hälften gespalten. Sie zwangen sie nach, ich glaube, Terezin zu gehen, aber sie setzte sich und sagte, mit ihrem klaffenden Kopf, ich gehe nicht, und der Schnee deckte sie zu. ${ }^{16}$ Unhintergehbar bleibt die Erinnerung an die Vertreibung und Vernichtung der Juden, die Erinnerung daran ein Mahnmal, dass wir zwar oft und gerne bleiben wollen, aber nur selten bleiben können. „Wir sind nur Gast auf Erden“, heißt es in einem alten Kirchenlied. 


\section{Schreiben in einem fremden Sprach- und Kulturraum}

11 George Tabori schreibt seine ersten Romane, die zwischen 1944 und 1951 in englischen und amerikanischen Verlagen erscheinen, auf Englisch und er fährt fort, auf Englisch zu schreiben, als er längst wieder in Deutschland lebt und auf deutschen Bühnen seine eigenen Stücke auf Deutsch inszeniert. Jene Stücke, auf denen vornehmlich der Ruhm Taboris beruht - „Die Kannibalen“, „Mein Kampf“, „Jubiläum“, „Die Ballade vom Wiener Schnitzel“ -, sind Stücke, die in expliziter Weise auf die deutsche Geschichte der Judenvernichtung rekurrieren und der Aufarbeitung dieses Kapitels deutschösterreichischer Geschichte gewidmet sind, die - deutlich erkennbar - in deutschen oder österreichischen Kontexten angesiedelt sind und in Gegenwart und Vergangenheit ausdrücklich auf diese Bezug nehmen.

Bereits im Hinblick auf diese Stücke ließe sich von der Situation des schreibenden Migranten sprechen, insofern Tabori ja als Mitfünfziger mit langjähriger Auslandserfahrung nach Deutschland zurückkehrt und aus dieser Außenseiterposition heraus seinen radikal anderen Blick auf Juden und Judentum praktiziert. Es ist nicht nur die Verdoppelung und Verdreifachung der Spielebenen - eine Privatperson spielt einen Schauspieler, der eine Bühnenfigur spielt -, die es Tabori ermöglicht, sich mit dem ihn auch privat-existentiell betreffenden Thema des Holocausts zu beschäftigen; hinzu kommt noch der zusätzliche Filter der fremden Sprache (in diesem Fall des Englischen). Tabori nutzt diese vielen Filter, um sich und uns, den Zuschauern, eine gewisse Distanznahme zu gestatten, ohne doch die subjektive „Betroffenheit" ${ }^{{ }_{17}}$ aufzugeben. Der Skandal, den Tabori mit seinen ,jüdischen“ Stücken erregte, war zumindest ein zweifacher: Zum einen rückte er vom Bild des Juden als eines unschuldigen Opfers ab und präsentierte Juden als habgierige, ängstliche, rachsüchtige, gewalttätige, bornierte und unbelehrbare Menschen - wie alle anderen eben -, zum anderen zielte er mit seinen „authentischen Rollenspielen "18 auf eine „kathartische“ ${ }^{\text {"19 }}$ Wirkung des Theaters auf die an einer Aufführung Beteiligten, auf eine Wirkung, die „unter die Haut gehe“. „Du bist engagiert wie wir alle, um das Wort Fleisch werden zu

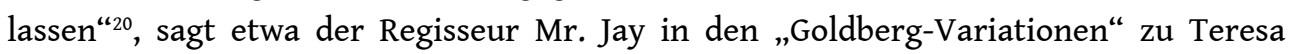
Tormentina Superstar und fokussiert damit auf jenen performativen und körperbezogenen Aspekt, der Taboris Theaterarbeit ausmacht. „Leere deinen Kopf, um die Welt der Haut zu empfangen “" ${ }^{21}$, heißt es ebendort. Nicht das theoretische Konzept, nicht die rationale Idee steht bei ihm im Vordergrund, sondern die Konzeption des Menschen - des Schauspielers wie des Zuschauers - als eines körperlichen, fleischlichen, körperlich empfindenden und reagierenden Wesens. Menschen (Schauspieler wie Zuschauer) müssen bei ihrer Leiblichkeit abgeholt und dort auch getroffen werden. „Ich weiß, dass keine Botschaft durchkommt, wenn sie nicht unter die Haut geht", sagt Tabori ${ }^{22}$. Deshalb landet Mr. Jays Assistent Goldberg, der eine tätowierte Lagernummer am Arm trägt, nicht zufällig am Ende des Stücks „Die Goldberg-Variationen“ als Stellvertreter Jesu auf dem Kreuz. Und die letzte Szene, in der sich die Figur Jesu mit jener des malträtierten Juden zusammenschließt, beginnt mit einer deutlichen Anspielung auf den Holocaust:

Mr Jay sitzt am Boden in einer Blutlache und spielt mit Goldbergs Schuhen. Mrs.

Mopp lugt durch den Vorhang ins (imaginäre) Publikum. 
MRS. MOPP Die Türen sind offen. Auf zu einem neuen Triumph. Aber mit zuviel Applaus würde ich nicht rechnen.

MR. JAY Was gibt's da zu applaudieren?

MRS. MOPP Ein Jid weniger. ${ }^{23}$

Der Widerspruch, den Tabori mit seinen Stücken zu Nazitum und Holocaust verursacht hat, ist sicherlich auch zum Teil dem Blick von außen zuzuschreiben, der dem Autor eine größere Unbefangenheit gegenüber der in Deutschland etablierten „Erinnerungsindustrie“ gewährte ${ }^{24}$. Es sei nicht sein Interesse, „deutsches Schuldgefühl zu manipulieren [...], dieses ganze sado-masochistische Verkleidungsspiel, das unsere Beziehung vergiftet, die offizielle Frömmigkeit, die all unseren Kummer und Haß und auch unsere Liebe verschleiert. Wenn wir“, so Tabori weiter, „nicht über die Tabus und Klischees hinwegsehen und einander als Menschen und nicht als Abstraktionen betrachten können, dann kann man genauso gut die Öfen wieder anzünden.“ ${ }^{25}$

Doch Tabori schreibt nicht nur Stücke zu Judentum, Holocaust und Nationalsozialismus (wenngleich diese Thematik in all seinen Texten mitschwingt), er thematisiert ebenso und hier ist ein weiterer Aspekt seiner eigenen Migrationserfahrung dingfest $\mathrm{zu}$ machen - die Ausgrenzungs-, Unterdrückungs- und Vernichtungsszenarien, die Zigeuner, Indianer oder Schwarze erfahren. In der Auseinandersetzung mit letzteren rekurriert Tabori auf spezifisch amerikanische Verhältnisse, die als potentiell ebenso gewalttätig, ungerecht, sadistisch erscheinen wie jene der Nazi-Schergen und NaziMitläufer.

14 „Die Demonstration“ heißt etwa ein Stück, das die Zuschauer in den Alltag des amerikanischen Rassismus entführt und demonstriert, wie Macht und Machtmissbrauch funktionieren. „Die Demonstration“ ist die (theatralische) Demonstration einer (politischen) Demonstration, die Inszenierung vielfältiger Schikanierungsmaßnahmen, denen Schwarze in den USA ausgesetzt sind. Wieder nutzt Tabori das Hilfsmittel der Potenzierung der Spielebenen, lässt also etwa die Schauspielerin mal als „sie selbst“, mal als „Schauspielerin“, mal als „Figur“ in einem Rollenspiel agieren und sprechen, lässt verschiedene Rollen und Rollenwechsel innerhalb eines Spiels zu. Gezeigt wird, wie Übergriffe von Sheriffs, Polizisten und Rechtsradikalen versuchen, dem Menschen, dem Schwarzen, seine Würde zu nehmen und wie an ihrer Brutalität jede Hoffnung auf ehrenhaften Widerstand zerbricht:

MONSIEUR Y Wenn ich versuche, mich denen gegenüber zu behaupten, komme ich mir vor - wie ein Schwindler. Das Pathos der Heldentaten, nehme ich an ... Kriecht herum Tut mir leid, Sie zu enttäuschen ... Die aufrechte Haltung ist mir natürlich lieber ... die eines Guerilla von Goya zum Beispiel ... Breitet die Arme aus ... wie er dem Exekutionskommando gegenübertritt. Bricht zusammen Aber um ehrlich zu sein, so kommt es einem viel wahrhaftiger vor ... ${ }^{26}$

Doch nicht nur die Gewalttätigkeit weißer Täter gegenüber schwarzen Opfern wird vorgeführt, sondern auch der Umschlag der Aggression geübt. Das geschieht innerhalb des Stücks zum einen durch den Wechsel zwischen den verschiedenen Rollen, im Zuge deren auch die beiden Schwarzen Weiße spielen - „und warum nicht? Opfer wissen Bescheid; sie kennen ihre Unterdrücker notgedrungen“27, heißt es. Zum andern manifestiert sich dieses mögliche Kippen aus der Opfer- in die Täterrolle z.B. auch in der subtilen Feindseligkeit der beiden Schwarzen auf der Handlungsebene des Stücks, wenn etwa der Schwarze Creampuff in die Teetasse seiner weißen Gastgeberin uriniert.

Gewaltbereitschaft bildet einen universellen Kontext, aus dem nur selten auszubrechen gelingt. Der Blick von außen, den der Kosmopolit und „Fremdling““28 George Tabori 
sowohl in Amerika wie auch in Europa trainiert, erlaubt ihm die schonungslose Offenlegung der Unterdrückungs- und Gewaltmechanismen, mit denen Schwächere, Außenseiter, sozial weniger Privilegierte weiter an den Rand gedrängt und dort festgehalten werden.

\section{Literatur als ästhetisches Mittel des Kampfes gegen Unterdrückung und Ausgrenzung}

Die Verzweiflung, die den meisten Stücken Taboris zugrunde liegt, erlaubt m.E. zwei Deutungen: Zum einen handelt es sich unleugbar um das private Trauma der Ermordung des Vaters im KZ, die den Anstoß zum Schreiben bildet und an die in fast allen Stücken in irgendeiner Weise erinnert wird. Im Interview fragt Tabori: „Jeder Sohn möchte irgendwann einmal seinen Vater umbringen; wenn aber - wie in meinem Fall - andere das für ihn erledigen, und er sich auf lähmende Weise zwischen einer Art von Erleichterung und dem heftigen Verlangen nach Rache schwanken fühlt - was dann? ${ }^{{ }^{2} 9}$

Zum andern geht es um eine sehr kritische Auseinandersetzung mit den Idealen der Aufklärung wie Toleranz, Freiheit, Gleichheit, Brüderlichkeit, Humanität, an denen festzuhalten als lächerliche Absonderlichkeit erscheint, deren Scheitern deutlich vor Augen geführt wird und deren Scheitern eben gerade für jene Verzweiflung verantwortlich ist, die Taboris Stücke so prägt. Beide Motivstränge sind insofern miteinander verknüpft, als es zumeist die Figur des Vaters ist, die nach wie vor für jene Ideale der Aufklärung eintritt und für sich einen Anspruch auf „Würde“ hochhält, der unter den unmenschlichen Bedingungen eines KZs nicht länger aufrechterhalten werden kann. Eindeutig steht dieser Konflikt in Taboris erstem „Europa“- Stück, den „Kannibalen“, im Mittelpunkt. Gewidmet ist dieses Stück bekanntlich seinem Vater, im Stück selbst ist es die Figur des Onkels, die den Part des Vaters übernimmt. Dessen Beharren auf Menschenwürde, sein Eintreten für Toleranz und Anstand, provoziert einen heftigen Konflikt mit den Mithäftlingen und macht sich, indem es einen durchaus möglichen Ausbruchsversuch vereitelt, auch „schuldig“. Sein Versuch, "Menschlichkeit" auch unter unmenschlichen Bedingungen zu bewahren, stößt sich also gleichermaßen an der Inhumanität der anderen wie an der implizierten Schuldfrage. Ist ein Mord zu rechtfertigen, wenn damit das Leben vieler Unschuldiger gerettet werden kann? Für den Onkel, der jede Gewalttätigkeit ablehnt, ist die Antwort eindeutig. Bei den anderen Mithäftlingen stößt diese Einstellung auf starken Widerstand.

RAMASEDER [zum Onkel] Dein Anzug am Tag unserer Verhaftung war höchst sonderbar.

ONKEL Es war mein Hochzeitsanzug.

RAMASEDER spöttisch Ins Gefängnis - mit einem Zylinder? Wen wolltest du denn heiraten?

ONKEL Es sollte eine Geste sein.

RAMASEDER Wie ist das zu verstehen?

ONKEL Es war ein festlicher Anlaß.

RAMASEDER Sie ließen dich den Bürgersteig aufwischen!

ONKEL Ja, eben, das meine ich ja. 
RAMASEDER Warum hast du sie nicht totgeschlagen? Onkel schweigt Und als die Männer dich holen kamen und dich nach draußen schleiften, durch die große Eingangstür, da hast du gesagt: Nach Ihnen, meine Herren. Ich hab mir verschiedenes aufgeschrieben, was du damals gesagt hast. Zum Beispiel hast du gesagt: „Die Gänse sind gekommen.“

ONKEL Ja.

RAMASEDER Du hast die Nazis gemeint?

ONKEL Ja.

RAMASEDER Warum hast du's dann nicht gesagt, verdammt noch mal? Onkel schweigt. Du hast auch gesagt: „Die einzige Methode, Gänsen zu widerstehen, ist die, einer Gans so unähnlich wie möglich zu bleiben." Was bedeutet das denn? Etwa weiße Handschuhe tragen? Eine Mahlzeit verfluchen? Hungern?

WEISS in den Topf hinein, so daß es wie aus großer Entfernung klingt Näch-sten-liebe! 30

18 Der Konflikt eskaliert, als die Mithäftlinge aus ihren Rollen fallen und - mitgerissen von der Dynamik der Erlebnisse - als Privatpersonen über den Schauspieler des Onkels herfallen.

Die Spieler treten aus dem Spiel heraus und zeigen die Brutalität der Unmenschen, die sie vorher nur dargestellt haben; für Augenblicke sind sie weder Väter noch Söhne, sondern haßerfüllte Fanatiker. Es wird privat gesprochen; Haas sagt: „Na, das war doch wohl nicht nötig“, oder ähnliches; der Zigeuner stürzt sich auf den Onkel, zerrt ihn hoch und kreischt: „Wisch den Fußboden auf, Itzig!“ Einige reagieren fassungslos erstaunt, man hört: „Seid ihr verrückt geworden?“ und „Um Gottes willen, hört doch auf" usw. ${ }^{31}$

Wie Macht funktioniert, wie Gewalt und Unterdrückung Hand in Hand gehen, wie aus Menschen - auch ehemaligen Opfern - Täter werden und warum es nicht helfen kann, den Menschen mit rationalen Argumenten zu Menschlichkeit „überreden“ zu wollen: davon handeln alle Stücke Taboris. Seine Intention ist es nicht, abstrakte Ideale, Dogmen, Axiome zu formulieren, sondern den Beweis der Fragilität dieser Glaubenssätze vor Augen zu führen. Nicht weil das Gute das Gute und dem Bösen überlegen ist, sondern weil gut und böse austauschbar sind, sich nur allzu leicht ineinander verkehren: eben darum bleibe dem Menschen nichts anderes übrig, als sich selbst und seiner Verführbarkeit $\mathrm{zu}$ Macht und Gewalt $\mathrm{zu}$ misstrauen und jene „authentische“, direkte Auseinandersetzung mit dem anderen zu suchen, die allein Verständnis garantiert. Wichtig scheint auch Taboris Erkenntnis, dass allzu offensichtliche Güte den weniger Guten nicht nur beschämt, sondern diesen auch dazu verleitet, seine moralische Unterlegenheit unter Beweis stellen zu wollen. „Ich glaube, Kain erschlägt den Abel, weil er dessen Güte nicht mehr ausstehen kann" “32. Unsinnig sei es, das Böse, Triebhafte, Elementare wegsperren zu wollen, es im Namen eines „höheren“ Ideals zähmen zu wollen. „Aber der Irrationalismus macht sich nicht davon, wenn wir ihn missbilligen; denn schließlich ist er Teil von jedem von uns" ${ }^{\text {"33. }}$. Unweigerlich verschaffe sich das Verdrängte, Nicht-Akzeptierte sein Recht. In dem Stück „Die 25. Stunde“ spricht Tabori von dem „Schmerz des Lebens“, dem wir uns zu stellen hätten, „und wenn wir diesen Schmerz nicht spüren, wenn wir ihn nicht schmecken, riechen, essen, leben, verwandelt er sich in einen kleinen Kannibalen, der sich durch unsere Lenden frißt ${ }^{\star{ }_{34}}$. Damit schließt Tabori scheinbar direkt an Adornos und Horkheimers Befund von der „Dialektik der Aufklärung“ an, die in der Wiederkehr des verdrängten Nicht-Rationalen die Ursache aller Katastrophen der Moderne sehen. 
„Jeder Versuch, den Naturzwang zu brechen, indem Natur gebrochen wird, gerät nur um so tiefer in den Naturzwang hinein “"35.

Nur wenigen Figuren ist bei Tabori so etwas wie „Unschuld“ gewährt, die sie vor Machtmissbrauch und Gewaltanwendung schützt. Zumeist handelt es sich dabei um Frauen und um Behinderte. Angela - nomen est omen - in der „Ballade vom Wiener Schnitzel“ ist eine davon oder Ruthie in "Weisman und Rotgesicht" oder Gretchen in „Mein Kampf“. Sie sind „naiv“ auf eine instinktiv-subtile Weise, der Natur verbunden und entziehen sich den Machtdemonstrationen ihrer Männer. Über Gretchen in „Mein Kampf" heißt es etwa:

Fräulein Gretchen Maria Carolina Globuschek-Bornemissza-Eszterfalvy ist nicht nur die letzte Jungfrau über vierzehn in Wien, sie ist sozusagen ein Wesen zwischen den Arten, ohne die evolutionäre Kluft zwischen dem Frosch und dem Homo sapiens. Sie ist noch Teil der Natur; wenn sie quakt, antworten die Enten, die Elefanten begrüßen sie mit einem Trompetenstoß, und man weiß von Zugvögeln, die ihr in die Wiener Wälder folgen, statt, wie geplant, nach Afrika zu ziehen. ${ }^{36}$

Im Unterschied dazu sind die Männerpaare zu sehen. Häufig sind Taboris Stücke um einen zentralen Machtkonflikt zwischen zwei Männern gruppiert, wobei der eine eindeutig das Sagen hat und der andere in seinem Dulden aufbegehrt: Mr. Jay und Goldberg, Weisman und Rotgesicht, Hitler und Herzl u.a.m. Im Kampf um Täterschaft und Opfertum sind sie aufeinander angewiesen. Häufig provoziert die moralische Übermacht des einen die physische Gewalt des anderen. Besonders deutlich tritt dieses Schema in den „Goldberg-Variationen“ zutage, in denen nicht nur Mr. Jay und sein Assistent Goldberg einander als Protagonisten gegenüber stehen, sondern in vielen Szenen ähnliche Konfliktkonstellationen nachgestellt werden: Moses und Aaron, Abraham und Isaak, Kain und Abel.

Nicht ein engagiertes Plädoyer für die schwächere Seite bietet Tabori an, sondern eine differenzierte Sicht der gegenseitigen Abhängigkeit von Täter und Opfer und ihrer potentiellen Austauschbarkeit. Nicht die Verdammung des Täters steht hier im Vordergrund, sondern der - letztendlich scheiternde - Versuch, Täterschaft $\mathrm{zu}$ verstehen und sie transzendental-moralisch zu rechtfertigen. „Wer sind wir denn, daß es uns erlaubt ist, uns mit dem Himmlischen zu beschäftigen?“" ${ }^{37}$, heißt es in „Die Ballade vom Wiener Schnitzel“.

Ausgrenzung und Unterdrückung erscheinen dann nicht als temporär oder periodisch auftretende Phänomene und Probleme einer spezifischen Gesellschaft, sondern als Konstanten menschlichen Verhaltens, deren Entstehung von bestimmten Umweltsachverhalten gefördert wird und deren Erfahrung „am eigenen Leib“ keinen Garanten für Immunisierung dagegen darstellt. Insofern sind Taboris Stücke auch sehr "moralisch“. Immer gilt es, sich der eigenen, individuellen Schuld zu stellen, denn - wie es in den „Goldberg-Variationen“ heißt - „niemand über fünf ist unschuldig“"38.

\section{Schluss}

„Ich habe keine Heimat, in jedem Sinn des Wortes Heimat, nicht einmal einen

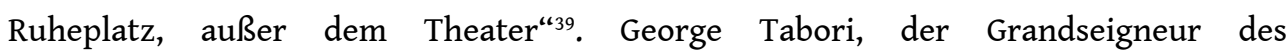
zeitgenössischen Theaters, erscheint sowohl im Hinblick auf seine Biographie wie auch auf die Themen seiner Texte als Paradebeispiel für Migrationsliteratur, der die Mühen der Migration, des Exils, des Schreibens in einem fremden Sprach- und Kulturraum und 
die Erfahrung von Unterdrückung und Benachteiligung als wesentliche Merkmale zugehören. Im Unterschied zu dem im Rahmen deutscher Migrationsliteratur häufig anzutreffenden gesellschaftskritischen Engagement, das die Vorurteile und Borniertheit einer deutschen Öffentlichkeit anprangert und für eine plurikulturelle Gesellschaft plädiert, stehen in Taboris Texten, vor allem in seinen Stücken, von denen hier vornehmlich die Rede ist, allgemein menschlich-moralische Überlegungen im Vordergrund. Nicht die Fremdheitserfahrung einer japanischen Intellektuellen in Deutschland, nicht die Marginalisierung eines griechischen oder türkischen Gastarbeiters in Deutschland und nicht die Ausgrenzung junger Afrikaner auf dem deutschen Arbeitsmarkt werden hier thematisiert, sondern das Problem der Täterschaft und der daran geknüpften Schuldfrage. Menschen werden zu Tätern, auch wenn sie Opfer waren, und manchmal werden sie zu Opfern, obgleich sie zuvor Täter waren, und sie werden dadurch schuldig am anderen. Sie werden schuldig am andern, weil sie ihn in seiner Eigenständigkeit und Menschenwürde nicht zu akzeptieren bereit sind, weil sie sich nicht auf eine unbefangene, offene Konfrontation mit diesem anderen einlassen, sondern lieber ihre Vorurteile und Klischees pflegen. Und sie tun dies, weil sie Menschen sind, d.h. eben keine Konstrukte abstrakter Konzepte oder theoretischer Absichtserklärungen, sondern Menschen aus Fleisch und Blut, gefangen in ihrer Haut, mit einer Angst, die in ihren Eingeweiden sitzt und sich als Scheiße realisiert. „Man kann nicht aus seiner Haut, und also habe ich in meiner Hose jetzt nicht nur meine Exkremente, sondern auch meine Empörung. Ich habe mich niemals menschlicher gefühlt" ${ }^{\text {*0 }}$.

Taboris Stücke stellen die immergleiche Frage nach der Möglichkeit oder Unmöglichkeit menschlichen, „humanen“ Handelns und sie geben im Allgemeinen keine Antwort, sondern bleiben zumeist im Habitus der Verzweiflung stecken. Nur manchmal taucht so etwas wie ein Schimmer von Heilshoffnung auf, wenn Versöhnung praktiziert wird - wenn etwa Angela und Morgenstern auf einem Friedhof mit verwüsteten jüdischen Gräbern eine Versöhnungsmahlzeit - mit Wiener Schnitzel! feiern:

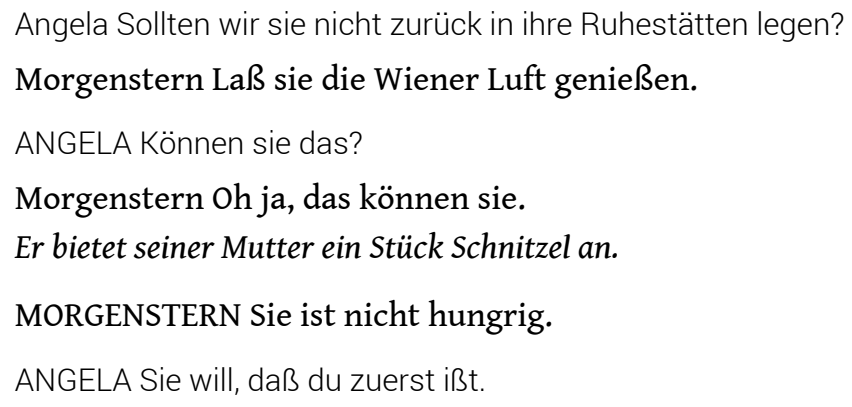

Das Pendeln zwischen verschiedenen Sprachen und Kulturen mag mit dazu beigetragen haben, dass Tabori seine ganz eigene Sicht auf Exil, Verfolgung, Benachteiligung entwickeln und präsentieren und zu einer Form des Theaters finden konnte, in der europäische Theatertradition und amerikanische Theaterschulen miteinander verschmelzen. Die Mehrfachverankerung in unterschiedlichen Kontexten ist eine wesentliche Voraussetzung für seine Form von „Weltliteratur“; sie bedeutet in der Radikalität ihrer Entstehung aber auch den Verzicht auf Heimat und Geborgenheit - 
oder wie Tabori einmal in einem Interview über das Problem der Zweisprachigkeit sagte: „Aber diese Zweisprachigkeit ist schwierig. Es gibt Beispiele: Beckett - Joseph Conrad, Nabokov - [Einwurf des Interviewers, A.B.] Julien Green, ja ... Wir sind ja alle Flüchtlinge, Fremde. Wir sind alle Ausländer auf dieser Welt ${ }^{\star 42}$.

\section{NOTES}

1. George Tabori: «'Ich war immer müde'. Gespräch mit Iris Radisch», in: Die Zeit, Nr. 22 (19. Mai 2004), S. 46.

2. A. Mansour Bavar: Aspekte der deutschsprachigen Migrationsliteratur. Die Darstellung der Einheimischen bei Alev Tekinay und Rafik Schami, München, Iudicium, 2004, S.46.

3. Gerhard Bauer: «Literarische Weltbürgerschaft und ihre Hindernisse», in: Aglaia Blioumi (Hg.): Migration und Interkulturalität in neueren literarischen Texten, München, Iudicium, 2002, S. 15-27, 26.

4. Heidi Rösch: Migrationsliteratur im interkulturellen Kontext. Eine didaktische Studie zur Literatur von Aras Ören, Aysel Özakin, Franco Biondi und Rafik Schami, Frankfurt/Main, Verlag für Interkulturelle Kommunikation, 1992, S. 12.

5. Tabori, «'Ich war immer müde'» [Anm. 1].

6. George Tabori: «Die Demonstration», in: Ders.: Theaterstücke I. Mit einem Vorwort von Peter von Becker. Aus dem Englischen von Ursula Grützmacher-Tabori, Frankfurt/Main, Fischer, 1996, S. 147-170, 152.

7. Zit. nach P. Iyer: «The Empire Writes Back. Am Beginn einer neuen Weltliteratur? », in: Neue Rundschau, Jg. 107 (1996), H. 1, S. 9-19.

8. Elisabeth Bronfen, Benjamin Marius: «Hybride Kulturen. Einleitung zur anglo-amerikanischen Multikulturalismusdebatte», in: Dies. (Hg.): Hybride Kulturen. Beiträge zur angloamerikanischen Multikulturalismusdebatte, Tübingen, Stauffenberg, 1997, S. 1-29, 18.

9. „Paradies verloren“ heißt nicht zufällig das neueste Buch des holländischen „global players“ Cees Nooteboom (Roman. Aus dem Niederländischen von Helga van Beuningen. Frankfurt/Main: Suhrkamp 2005).

10. George Tabori: Tod in Port Aarif. Roman. Aus dem Englischen von Ursula Grützmacher-Tabori. Hg. und mit einem Nachwort von Wend Kässens, Göttingen, Steidl, 2004, S. 25.

11. Ebd., S. 11.

12. George Tabori: «Mein Kampf», in: Ders.: Theaterstücke II. Deutsch von Ursula GrützmacherTabori. München, Wien, Hanser, 1994, S. 143-203, 155.

13. George Tabori: «Die Ballade vom Wiener Schnitzel. Deutsch von Ursula Grützmacher-Tabori», in: Theater heute, 1996, H. 5, S. 46-52, 48.

14. George Tabori: «Weisman und Rotgesicht», in: Ders.: Theaterstücke II. Deutsch von Ursula Grützmacher-Tabori, München, Wien, Hanser, 1994, S. 205-239, $234 f$.

15. Nicht zufällig bilden deswegen auch die Themen Judentum, Judenverfolgung, das Bild des Juden u.ä. zentrale Themen der Tabori-Forschung, z.B. Thomas Rothschild: «Die Wunde versteht das Messer. Juden auf Taboris Bühne», in: text + kritik, 1997, Nr. 133, S. 4-9; Anat Feinberg-Jütte: «Erinnern, Wiederholen, Durcharbeiten. George Taboris Jubiläum'», in: text + kritik, 1997, Nr. 133, S. 71-80; Jan Strümpel: Vorstellungen vom Holocaust: George Taboris Erinnerungs-Spiele, Göttingen, Wallstein-Verlag, 2000. [Göttingen, Diss. 1999]

16. Tabori, «Die Ballade vom Wiener Schnitzel» [Anm. 13], S. 52. 
17. Besonders Franco Biondi und Rafik Schami haben das Verständnis von der „Migrationsliteratur" als einer „Literatur der Betroffenheit“ favorisiert. Vgl. dazu Franco Biondi, Rafik Schami: «Literatur der Betroffenheit. Bemerkungen zur Gastarbeiterliteratur», in: Christian Schaffernicht (Hg.): Zu Hause in der Fremde, Reinbek bei Hamburg, Rowohlt, 1984, S. 136-150.

18. Diese geniale Bezeichnung stammt von Hajo Kurzenberger, der damit die ambivalente Zwitterstellung Taboris zwischen den Theaterkonzepten von Bert Brecht und Lee Strasberg auf den Punkt bringt. Hajo Kurzenberger: «Taboris authentische Rollenspiele», in: text + kritik, 1997, Nr. 133, S. 58-70.

19. Vehement protestiert er im Geleitwort zur europäischen Erstaufführung der „Kannibalen“ gegen ,jene, die dem Theater seine Urfunktion absprechen möchten, nämlich Furcht und Erbarmen zu erzeugen und auf mythisch-zeremoniöse Art jene Gemeinsamkeit zu schaffen, auf deren Boden alles verhöhnt werden kann, was als heilig gilt, und sei es nur, um zu entdecken, was davon noch Gültigkeit besitzt.“ George Tabori: «Die Kannibalen. Zur europäischen Erstaufführung», in: Ders.: Unterammergau oder Die guten Deutschen, Frankfurt/Main, Suhrkamp, 1981, S. 37-39, 37.

20. George Tabori: «Die Goldberg-Variationen», in: Ders.: Theaterstücke II. Aus dem Englischen von Ursula Grützmacher-Tabori, München, Wien, Hanser, 1994, S. 291-346, 309.

21. Ebd., S. 311.

22. George Tabori: «Wenn die Leute vom Theater reden ... ", in: Andrea Welker (Hg.): George Tabori. Dem Gedächtnis, der Trauer und dem Lachen gewidmet, Wien, Linz, Weitra, München, Bibliothek der Provinz, 1994, S. 25-32, 29.

23. Tabori, «Die Goldberg-Variationen» [Anm. 20], S. 344.

24. Vgl. Taboris Skepsis gegenüber Auschwitz selbst: „Auschwitz ist ein Museum. Es gibt dort nichts Lebendiges, nichts, an dem sich Gefühle entzünden könnten. Auschwitz ist sehr touristisch, viele Japaner. Ich habe in Auschwitz verzweifelt den Geist meines Vaters gesucht und nicht gefunden.“ „Interview von Sven Michaelsen mit George Tabori und Claus Peymann: Gipfeltreffen der Provokateure“, in: Stern, H. 22 (26.5.1994), S. 62-70, 70.

25. George Tabori: «Unterammergau oder Die guten Deutschen», in: Ders.: Unterammergau oder Die guten Deutschen, Frankfurt/Main, Suhrkamp, 1981, S. 7-28, 24.

26. Tabori, «Die Demonstration» [Anm. 6], S. 164.

27. Ebd., S. 155.

28. So bezeichnete sich George Tabori selbst in einem Gespräch, das vor ein paar Jahren vom ORF im Rahmen der Sendung „Einfach klassisch“ gesendet wurde. Leider war es mir nicht mehr möglich, das genaue Datum zu eruieren.

29. Tabori, «Die Kannibalen. Zur europäischen Erstaufführung» [Anm. 19], S. 37.

30. George Tabori: «Die Kannibalen», in: Ders.: Theaterstücke I. Mit einem Vorwort von Peter von Becker. Deutsch von Peter Sandberg, Frankfurt/Main, Fischer, 1996, S. 1-74, 39.

31. Ebd., S. 64.

32. „Solche Begegnungen habe ich mein Leben lang gefürchtet und mir gewünscht“. «Gespräch mit George Tabori von Ursula Voss und Peter von Becker», in: Theater heute, 1990, H. 5, S. 16-18, 18.

33. «Ein begeisterungsfähiger Skeptiker. Gespräch von Rolf Michaelis mit dem Autor und Regisseur George Tabori», in: Theater heute, 1976, H. 6, S. 29-33, 33.

34. George Tabori: «Die 25. Stunde», in: Ders.: Theaterstücke I. Mit einem Vorwort von Peter von Becker. Deutsch von Ursula Grützmacher-Tabori, Frankfurt/Main, Fischer, 1996, S. 231-283, 264.

35. Max Horkheimer, Theodor W. Adorno: Dialektik der Aufklärung. Philosophische Fragmente, Frankfurt/Main, Fischer, 1985, S. 15.

36. Tabori, «Mein Kampf» [Anm. 12], S. 153.

37. Tabori, «Die Ballade vom Wiener Schnitzel» [Anm. 13], S. 52.

38. Tabori, «Die Goldberg-Variationen» [Anm. 20], S. 327. 
39. «Ein begeisterungsfähiger Skeptiker. Gespräch von Rolf Michaelis mit dem Autor und Regisseur George Tabori» [Anm. 33], S. 32.

40. Tabori, «Die Demonstration» [Anm. 6], S. 167; es gibt viele andere ähnliche Stellen in Taboris Werk, die auf die Rolle der Haut, ihre Abschirmfunktion und ihre Verletzlichkeit Bezug nehmen.

41. Tabori, «Die Ballade vom Wiener Schnitzel» [Anm. 13], S. 52.

42. «Diese große Lebensreise. George Tabori: Zeuge des Jahrhunderts - im Gespräch mit Peter von Becker», in: Theater heute, 1994, H. 5, S. 8-17, 17.

\section{RÉSUMÉS}

Der Artikel behandelt das Werk des ungarnstämmigen, jüdischen, auf Englisch schreibenden und seit langem auf deutschen Bühnen inszenierenden Kosmopoliten, Emigranten und „Fremdlings“ George Tabori. Drei Aspekte der Migrationsliteratur werden genauer untersucht: 1) das Thema der Migration, 2) Schreiben in einem fremden Sprach- und Kulturraum, 3) literarisch-ästhetische Strategien des Kampfes gegen Unterdrückung und Ausgrenzung. Alle drei Aspekte spielen in Taboris Werk eine wichtige Rolle. Eine genaue Analyse zeigt jedoch, dass bei Tabori Phänomene und Probleme der Migration und der Migrationsliteratur unter dem Fokus der Frage nach „richtigem“ menschlichen Handeln zusammengefasst werden. Tabori interessiert sich dafür, wie aus Opfern Täter werden - und umgekehrt. Seine Problemstellung ist eine zutiefst moralische und belässt es zumeist bei der Verzweiflung über die Unausweichlichkeit des Menschen, schuldig zu werden.

Nous étudierons dans cette contribution l'œuvre de l'auteur juif George Tabori, originaire de Hongrie, qui écrit en anglais et met depuis longtemps en scène, en Allemagne, des personnages cosmopolites, des migrants et des «étrangers ». Nous étudierons plus précisément trois aspects de la littérature de la migration: 1) le thème de la migration, 2) l'écriture dans un contexte culturel et linguistique étranger, 3) les stratégies littéraires et esthétiques pour lutter contre l'oppression et la marginalisation. Ces trois aspects jouent dans l'œuvre de Tabori un rôle important. Nous découvrirons néanmoins que les questions de la migration et de la littérature de la migration, chez Tabori, se résument à la question de savoir quelle attitude adopter en tant qu'être humain. Ce qui intéresse Tabori, c'est de savoir comment, de victime, on devient bourreau - et inversement. La problématique ainsi posée relève essentiellement de la morale et traduit la désespérante vocation de l'individu à se rendre coupable.

\section{INDEX}

Mots-clés : Hongrie, émigration hongroise, littérature de la migration

oeuvrecitee Mein Kampf, Die Ballade vom Wiener Schnitzel, Die Goldberg-Variationen

\section{AUTEURS}

\section{ALICE BOLTERAUER}

Universität Graz 